Acta Poetica $33 \bullet 1$

ENERO-JUNIO

$2012(67-85)$

\title{
Credibilidad e intertextualidad: el ethos del buen testigo en la Recherche de Proust
}

\author{
Pierre Zoberman
}

Este artículo analiza la manera como las conexiones intertextuales, en particular con los textos del siglo XVII, pero también intratextualmente con la novela misma, ayudan a crear un ethos del testigo fidedigno en la Recherche de Proust. Proust explícitamente desarrolla la teoría de que el escritor provee al lector de unos lentes especiales para ver la realidad que el escritor reconstruye con un relieve particular. Su Narrador exhibe poderes inusuales de observación — percibiendo, por ejemplo, una "imperceptible" mancha roja en la corbata de Charlus en su primer encuentro - lo que le permite dar una explicación de primera mano de la desintegración del aristocrático Faubourg-Saint-Germain, erróneamente visto, en un principio, como perenne e inmutable, por lo tanto, esencial. Ya sea como resultado de la ingenuidad (cuando el Narrador no entiende lo que ve) o de un afán de saberlo todo (una vez que logra comprender y que comparte un sorprendente conjunto de 'hechos' con el lector), la novela vuelve a destacar la fiabilidad del testimonio del Narrador en relación con la homosexualidad, tema de creciente significado. Pero este ethos, ostensiblemente basado en una observación de primera mano, está reforzado, si no es que totalmente construido, por una compleja relación con las Cartas de Sévigné o las Memorias de Saint-Simon, que pueden considerarse no solo como fuentes o referencias, lo que algunos críticos han ampliamente enfatizado, sino como textualmente produciendo (generando) pasajes significativos de la Recherche. Esta estrategia intertextual es enteramente dominante, dado que el Narrador mismo delega sus afanosos poderes de observación en otros personajes, cuyas propias observaciones dependen, ellas mismas, de conexiones textuales, esta vez, mayoritariamente intratextuales con la Recherche misma —empleando, de nuevo, procesos intertextuales para crear la caracterización.

Palabras Clave: Proust, ethos/caracterización, intertextualidad, homosexualidad, colores. 
This article analyzes the way intertextual connections, especially to 17th-century texts but also intratextually to the novel itself, help create an ethos of the truthful witness in Proust's Recherche. Proust explicitly develops the theory that the writer provides readers with special glasses to see the reality which the writer recasts with special relief. His Narrator exhibits unusual powers of observation - perceiving, for instance, an "imperceptible" spot of red on Charlus's cravat at their first meeting - enabling him to give a first-hand account of the disintegration of the aristocratic Faubourg-Saint-Germain, mistakenly seen at first as perennial and immutable, hence essential. Whether it be the result of naïveté (when the Narrator does not understand what he sees) or knowingness (once he has become enlightened and shares a surprisingly large store of 'facts' with the reader), the novel again foregrounds the reliability of the Narrator's testimony with regard to homosexuality, a theme of ever-increasing significance. But this ethos, ostensibly based on first-hand observation, is reinforced, if not wholly constructed, by a complex relationship with Sévigné's Letters or SaintSimon's Memoirs, which can be shown, not only to be thematic sources or references, something critics have long emphasized, but textually to produce (to generate) significant passages of the Recherche. This intertextual strategy is all pervasive, since the Narrator himself delegates his keen powers of observation to other characters, whose own observations are themselves dependent on textual connections, this time mostly intratextual, to the Recherche itself - again using intertextual processes to create characterization.

KeYwords: Proust, ethos/characterization, intertextuality, homosexuality, colors.

Fecha de recepción: 31 de octubre de 2011

Fecha de aceptación: 13 de mayo de 2012 
Pierre Zoberman

Université Sorbonne Paris Cité - Paris 13

Centre d'Études et de Recherches Comparatistes, Francia

\section{Credibilidad e intertextualidad: el ethos del buen testigo en la Recherche de Proust}

[Traducción del inglés de Víctor Manuel Godínez Zúñiga]

Proust está lejos de ser el primero — o el único- autor en hacer la crónica de la desaparición de una elite privilegiada, elite que dejó el lugar a una nueva clase dirigente para la cual el dinero reemplazó los méritos de cuna. En Proust, como en Edith Wharton, las maneras de los arribistas traicionan su reciente ascensión a la capa superior de la sociedad, mientras que el cortesano en la corte de Louis XIV pudo haber aprendido de Faret (L'Honneste homme ou l'art de plaire à la court), o del vasto acervo de manuales de civilidad que acumuló el siglo XVII, cómo comportarse para ser uno de los honnêtes gens que crearon ambientes educados y mondains. A pesar del número siempre creciente de burgueses que alcanzaron los más altos escalones y lograron mezclarse exitosamente con los miembros de la elite aristocrática, Molière o La Bruyère arrojaron luces reveladoras del comportamiento característico de los financieros y nouveaux riches, de la misma manera en que Edith Wharton mostró la disolución del viejo Nueva York y el surgimiento de nuevos parámetros del éxito y el poder. ${ }^{1}$

${ }^{1}$ Véase, por ejemplo, The Custom of the Country o incluso The House of Mirth, en donde el personaje de Simon Rosedale combina lo nuevo rico con lo judío. 
La manera como Proust se erige en el cronista de un cambio de era consiste en crear a un Narrador dotado de amplios poderes de observación, o al menos así pareciera. ${ }^{2}$ Tal es el ethos de perceptibilidad superlativa que intento explorar aquí. El autor de la Recherche se esfuerza en persuadir al lector de que su Narrador es, en efecto, un personaje perspicaz y enfatiza las percepciones: el Narrador es representado como alguien concentrado en los signos, ${ }^{3}$ principalmente visuales pero también auditivos. La finura de observación está puesta de relieve en el texto no solo por medio de pasajes que afirman explícitamente que el verdadero escritor permite enfocar (como si fuera un instrumento óptico) la visión de sus lectores o el hecho de que tal o cual personaje esté dotado de grandes cualidades de observación, sino también, a nivel de la estructura narrativa, por medio de la narrativización de escenas de observación, ya sea que se trate del Narrador observando a Albertine dormida, o de diversas escenas de voyeurismo, o también a través de conversaciones sorpresivas durante encuentros fortuitos o en episodios en los que se espía y se presta oídos voluntariamente. En las reflexiones que siguen pretendo explicar la estrategia particular que consiste en legitimar intertextualmente secuencias que dependen ostensiblemente de la exactitud de la observación directa, visual o de otra naturaleza —en otras palabras, utilizando la intertextualidad para producir una caracterización ética. De hecho, esta estrategia está de alguna manera oscurecida a nivel textual por el énfasis explícito que mencioné anteriormente sobre la capacidad que poseen los (buenos) escritores de proveer a sus lectores de potentes lentes que les permitan observar aquello que normalmente no verían o malinterpretarían.

El hecho de que el Narrador se habilita a sí mismo como un observador particular y, con ello, fortalece su credibilidad como un testigo fidedigno, queda claro en citas como la siguiente, que rebela su recién encontrada pericia en el ámbito de la identidad (homo)sexual:

\footnotetext{
${ }^{2}$ De ahí la irónica observación de Compagnon en "La Dernière Victime" sobre la ceguera del Narrador cuando se trata de la homosexualidad, hasta que descubre la genuina identidad de Charlus ("c'[...]était une [femme]"). Véase más abajo De vuelta con el Narrador. De hecho, el Narrador está jugando con "ceguera y perspicacia", dialéctica que Paul de Man exploró hace algunas décadas.

${ }^{3}$ Como Deleuze ya lo enfatizó en su estudio decisivo Proust et les signes.
} 
[J]'entendis une voix d'une sorte qu'à l'avenir, je devais, sans erreur possible, discerner. C'était, dans le cas particulier, celle de M. de Vaugoubert causant avec M. de Charlus. Un clinicien n'a même pas besoin que le malade en observation soulève sa chemise ni d'écouter la respiration, la voix suffit. Combien de fois plus tard fus-je frappé dans un salon par l'intonation ou le rire de tel homme, qui pourtant copiait exactement le langage de sa profession ou les manières de son milieu, affectant une distinction sévère ou une familière grossièreté, mais dont la voix fausse suffisait pour apprendre; "C'est un Charlus" à mon oreille exercée comme le diapason d'un accordeur! (III, 63, énfasis agregado).

Las variantes recurrentes de la frase "je fus frappé", ${ }_{4}^{4}$ presentes cada vez que el Narrador introduce una observación que enfatiza como significante, refuerzan este ethos. Y lo refuerza a pesar de que, en realidad debido a que, el Narrador acaba siendo impresionado por algo apenas perceptible - otro medio recurrente, indirecto, de caracterizarse a sí mismo como un agudo observador-, con la paradoja adicional de que, en lugar de los detalles ahí presentes, susceptibles de ser mencionados por este observador acucioso, emergen ciertas semejanzas o rasgos específicos debido a que el Narrador y algunos otros personajes, apoyándose en sus ethos, ponen por delante un detalle como observable o revelador. Esta recurrencia será todavía más significativa al leerla en conexión con los ecos y las repeticiones intratextuales. ${ }^{5}$

\section{De Charlus como Moralista al Narrador como Memorialista}

Comenzaré por un pasaje en el que el hechicero intertextual no es el Narrador, a fin de mostrar hasta qué punto Proust favorece la intertextualidad como modo de producción textual. Apenas después de que se encuentra con Charlus por primera vez, el Narrador lo escucha lamentar

\footnotetext{
${ }^{4}$ Véase, por ejemplo, en relación con Saint-Loup, “je fus frappé combien il changeait" (IV, 281), o, hablando de Gilberte, "Et, en effet, j'avais été frappé d'une vague resemblance qu'on pouvait à la rigueur trouver maintenant entre elles. Peut-être tenait-elle à $[\ldots]$ quelques traits (dus par exemple à l'origine hébraïque pourtant si peu marquée chez Gilberte) [...]" (IV, 280).

${ }^{5}$ Véase más abajo Una estrategia proliferante.
} 
el hecho de que la residencia que alguna vez perteneció a una rama de la familia Guermantes haya sido recientemente adquirida por el financiero Israël. A primera vista el cliché antisemita de Charlus podría simplemente parecer como una observación realista, como un esmerado detalle que refuerza la verosimilitud de un personaje extraordinario - en otras palabras, como un mero factor en una estrategia de caracterización. En realidad, este pasaje está saturado de conexiones intertextuales:

Il raconta qu'une demeure qui avait appartenu à sa famille, où Marie-Antoinette avait couché, dont le parc était de Le Nôtre, appartenait maintenant aux riches financiers Israël, qui l'avaient achetée. "Israël, du moins c'est le nom que portent ces gens, qui me semble un terme générique, ethnique, plutôt qu'un nom propre. On ne sait pas, peut-être que ce genre de personnes ne portent pas de noms et son seulement désignées par la collectivité à laquelle elles appartiennent. Cela ne fait rien! Avoir été la demeure des Guermantes et appartenir aux Israëls!!! s'écria-t-il. Cela fait penser à cette chambre du Château de Blois où le gardien qui le faisait visiter me dit: "C'est ici que Marie Stuart faisait sa prière; et c'est là maintenant où ce que je mets mes balais." Naturellement je ne veux rien savoir de cette demeure qui s'est déshonorée, pas plus que de ma cousine Clara de Chimay qui a quitté son mari. Mais je conserve la photographie de la première encore intacte, comme celle de la princesse quand ses grands yeux n'avaient de regards que pour mon cousin. La photographie acquiert un peu de dignité qui lui manque, quand elle cesse d'être une reproduction du réel et nous montre des choses qui n'existent plus. Je pourrai vous en donner une, puisque ce genre d'architecture vous intéresse", dit-il à ma grand-mère (II, 123).

La mención de Le Nôtre podría leerse como una señal de que el siglo XVII encierra la clave del pasaje precedente. En efecto, esta clase de indicios de decadencia pueden encontrase en los escritos moralistas del siglo XVII. Zénobie, en "Des biens de fortune" (78) presenta un caso muy similar:

Ni les troubles, Zénobie, qui agitent votre empire, ni la guerre que vous soutenez virilement contre une nation puissante depuis la mort du roi votre époux, ne diminuent rien de votre magnificence. Vous avez préféré à toute autre contrée les rives de l'Euphrate pour y élever un superbe 
édifice: l'air y est sain et tempéré, la situation en est riante; un bois sacré l'ombrage du côté du couchant; les dieux de Syrie, qui habitent quelquefois la terre, n'y auraient pu choisir une plus belle demeure. La campagne autour est couverte d'hommes qui taillent et qui coupent, qui vont et qui viennent, qui roulent ou qui charrient le bois du Liban, l'airain et le porphyre; les grues et les machines gémissent dans l'air, et font espérer à ceux qui voyagent vers l'Arabie de revoir à leur retour en leurs foyers ce palais achevé, et dans cette splendeur où vous désirez de le porter avant de l'habiter, vous et les princes vos enfants. N'y épargnez rien, grande Reine; employez-y l'or et tout l'art des plus excellents ouvriers; que les Phidias et les Zeuxis de votre siècle déploient toute leur science sur vos plafonds et sur vos lambris; tracez-y de vastes et de délicieux jardins dont l'enchantement soit tel qu'ils ne paraissent pas faits de la main des hommes; épuisez vos trésors et votre industrie sur cet ouvrage incomparable; et après que vous y aurez mis, Zénobie, la dernière main, quelqu'un de ces pâtres qui habitent les sables voisins de Palmyre, devenu riche par les péages de vos rivières, achètera un jour à deniers comptants cette royale maison, pour l'embellir, et la rendre plus digne de lui et de sa fortune (La Bruyère, 202).

Pero por más semejantes que ambos pasajes puedan parecer, aún nos encontramos en el nivel del marco de referencia o del contexto (algo similar, por ejemplo, a la lectura de Noémi Hepp de La Bruyère como fuente de las meditaciones sobre el amor que se desarrollan en la Recherche) ${ }^{6}$ Charlus define sus propios gustos por medio de referencias a fuentes del siglo XVII, las cartas de Sévigné y Andromaque y Phèdre de Racine; y para que el Narrador cumpla su tarea de observador, Proust necesita evocar libros del pasado. En su "búsqueda del tiempo perdido", Proust recurre en particular a Saint-Simon cuando lleva al mismo Charlus, que hasta ahora ha sido solo un nombre, al interior del texto, esto es, en presencia del Narrador mismo... Y aunque las memorias de SaintSimon adquieren una mayor significancia en la Recherche a medida que el proyecto de Proust se desarrolla gradualmente, el diálogo entre ambos trabajos resultó en un muy productivo proceso intertextual.

\footnotetext{
${ }^{6}$ Ver Noémi Hepp, "Le XvII siècle de Marcel Proust dans La Recherche du temps perdu".
} 
La totalidad del pasaje donde el Narrador ve a Charlus por primera vez sin saber quién es, y en donde el misterio es el tema central, está configurado para caracterizarlo como alguien confundido. ${ }^{7}$ También lo convierte en un agudo observador capaz de ver lo que no es visible. Lo que aquí me interesa es el hecho de que la perceptibilidad del Narrador se crea a través de una motivación intertextual. En otras palabras, hay un artificio por medio del cual se pretende que las cualidades que el Narrador aparenta tener sean creíbles, no debido a sus propias afirmaciones explícitas o a las de Proust acerca del ethos del primero, como las de un testigo fiable y por consiguiente creíble, sino porque están respaldadas por referencias intertextuales tanto como, o más que, por las declaraciones en sí mismas o por "auténticos" detalles hábilmente presentados en la novela.

Charlus, uno de los personajes más sobresalientes, aparece como el arquetipo de un amante de los colores cuyo gusto es suprimido por razones que aún no han sido reveladas. Pero la supresión misma la infiere el Narrador, quien, con base en un par de pistas, de las que una, al menos, literalmente se presenta como imperceptible, es capaz de descifrar, en su primer encuentro, una faceta de la personalidad del extraño, el aún no identificado Charlus, que solo se le esclarecerá por completo al inicio de Sodome et Gomorrhe, pero que ya está presente intertextualmente. En otras palabras, aunque sea de manera ostensible, el Narrador verá finalmente la verdad solo en retrospectiva. En efecto, Proust programó su despliegue en su primera presentación de Charlus.

Et la sobriété qu'ils [sus ropas] laissaient paraître semblait de celles qui viennent de l'obéissance à un régime, plutôt que du manque de gourmandise. Un filet de vert sombre s'harmonisait, dans le tissu du pantalon, à la rayure des chaussettes avec un raffinement qui décelait la vivacité d'un goût maté partout ailleurs et à qui cette seule concession avait été faite

${ }^{7}$ El Narrador, cuya función es, como antes se mencionó y siguiendo una de las teorías que la novela aplica, desarrollar lentes especiales, no puede él mismo interpretar el sentido de la mirada o de las ojeadas de otras personas (de ahí que malinterprete las "œillades" tanto de Gilberte como de Charlus). 
par tolérance, tandis qu'une tache rouge sur la cravate était imperceptible comme une liberté qu'on n'ose prendre (II, 112).

En estas páginas hay al menos dos anotaciones de supresión de color. ${ }^{8}$ Esta primera es especialmente reveladora por el hecho de que la contradicción implicada (el texto menciona, y por lo tanto el Narrador ve, una imperceptible mancha roja) puede reducirse, su significado iluminarse y el pasaje justificarse como intertextualmente producido si observamos que Proust/el Narrador está jugando con el retrato de Monsieur ${ }^{9}$ trazado por Saint-Simon:

C'était un petit homme ventru monté sur des échasses tant ses souliers étaient hauts, toujours paré comme une femme, plein de bagues, de bracelets, de pierreries partout, avec longue perruque toute étalée en devant, noire et poudrée, et des rubans partout où il en pouvait mettre, plein de toutes sortes de parfums, et en toutes choses la propreté même. On l'accusait de mettre imperceptiblement du rouge (Mémoires, II, 16, énfasis agregado),

acusación que se podría extender a Proust, quien provee a Charlus del rojo imperceptible que encontró en el retrato descrito por Saint-Simon. Incluso en Saint-Simon el rouge es habladuría venenosa, un rumor sin fundamento, un discurso referido, no es conocimiento ni una certeza definitiva. Una vez más el texto erige al Narrador en un extraordinario observador, capaz de ver lo que no está ahí, o más bien lo que no puede verse. Pero el ojo perspicaz es el del lector, y Proust crea un retrato tomando de Saint-Simon el color que según él es suprimido en y por Charlus. Y en Charlus la supresión de los colores se vincula, en con-

${ }^{8}$ La segunda es tan reveladora, literalmente, como la primera: “À ce moment, apercevant que le mouchoir brodé qu'il avait dans sa poche laissait dépasser des lisérés de couleur, il le rentra vivement avec la mine effarouchée d'une femme pudibonde mais point innocente dissimulant des appas que, par un excès de scrupule, elle juge indécents" (II, 123).

9 "Monsieur" era el título que se le daba al hermano del Rey. El hermano de Louis XIV era conocido por sus relaciones homoeróticas exclusivas (con excepción de sus dos esposas) y Saint-Simon veía en ello la causa de los desórdenes jerárquicos. De ahí su retrato. 
traste, con su refinamiento, de la misma manera como Monsieur es "la propreté même", en otras palabras, el compendio del refinamiento.

Lo relevante aquí no solo es la conexión entre las dos figuras de Charlus y de Monsieur: todo un conjunto de lectores, desde De Ley hasta Jullien estableció el paralelo. Pero todos ellos tendieron a resaltar las conexiones temáticas/semánticas y no lograron ver los auténticos procesos intertextuales de engendramiento - la verdad del texto de Proust que literalmente depende del texto de las Mémoires, actualizándolo con sus propias palabras. La consternación de De Ley, al desarrollar el paralelo entre Monsieur y Charlus enfatizando la creciente propensión del segundo a usar maquillaje (y los desastrosos efectos ridículos que tiene) es paradigmática:

Nous nous passerions de relever un autre point de rencontre entre le baron et le frère du Roi, qu'on accusait de mettre imperceptiblement du rouge, si pendant toute une partie d'À la recherche du temps perdu, le leitmotiv du baron n'était pas précisément ses essais imparfaits de maquillage. Le baron, qui est dans les premiers volumes surtout "susceptible" revient dans Sodome et Gomorrhe fardé et grotesque comme un comédien de Callot, enfin c'est Brichot qui fait la remarque, poudré à son habitude (De Ley, Marcel Proust, 60)..$^{10}$

Para De Ley, en otras palabras, Proust se identifica con Saint-Simon, lo que significa que lo lee correctamente: "la critique a trop peu montré combien chez Saint-Simon aussi l'amour est source de peines et de souffrances" (56). ${ }^{11}$

${ }^{10}$ De Ley ya notó la devoción de Charlus como un rasgo inusual entre los personajes de Proust, enfatizando, de esta manera, la conexión con Monsieur como el origen del retrato de Proust.

${ }^{11}$ En el mismo sentido, Dominique Jullien muestra que Charlus, quien comparte la actitud religiosa de Monsieur, se asemeja cada vez más a este último conforme avanza la novela: "Le 'modèle' essentiel est bien sûr Monsieur, le frère du roi: Charlus lui doit son essence, son secret, l'inversion. Mais il hérite aussi, sinon de sa coquetterie enrubannée, du moins d'une certaine pusillanimité dans les questions spirituelles". Después de citar el retrato de Monsieur por Saint-Simon, Jullien afirma, en una nota: "Charlus, bien que gros comme Monsieur, se distingue au contraire par la sévérité de sa mise. Toutefois avec le temps qui amène sa déchéance, les fards seront chez lui de plus en plus perceptibles" (Proust et ses modèles, 68). El mismo Proust está deseoso de presen- 
Si el lector requiriera una confirmación de la estrategia textual que funda la credibilidad en los vínculos intertextuales con el siglo XVII, puede fácilmente obtenerla en la caudalosa tradición sobre la tante ${ }^{12}$ que el Narrador hace girar en torno a los precedentes históricos. Cuando escribe acerca de Madame de Vaugoubert como la encarnación de "la femme d'une tante" (llevando los pantalones en su casa, mientras su esposo es un Charlus por excelencia, como lo deja ver la cita al principio de este trabajo), alude a la imagen de Monsieur, hermano de Louis XIV y de su esposa, la princesa Palatine:

Mme de Vaugoubert réalisait le type, acquis ou prédestiné, dont l'image immortelle est la princesse Palatine, toujours en habit de cheval et qui, ayant pris de son mari plus que la virilité, épousant les défauts des hommes qui n'aiment pas les femmes, dénonce dans ses lettres de commère les relations qu'ont entre eux tous les grands seigneurs de la cour de Louis XIV. Une des causes qui ajoutent encore à l'air masculin des femmes telles que Mme de Vaugoubert est que l'abandon où elles sont laissées par leur mari, la honte qu'elles en éprouvent, flétrissent peu à peu chez elles tout ce qui est de la femme. Elles finissent par prendre les qualités et les défauts que le mari n'a pas. Au fur et à mesure qu'il est plus frivole, plus efféminé, plus indiscret, elles deviennent comme l'effigie sans charme des vertus que l'époux devrait pratiquer (III, 47).

Pero, una vez más, la referencia explícita a Madame y Monsieur funciona, a la vez, como señal y pantalla — señal para el lector que toma en consideración la caracterización de Saint-Simon de la pareja principesca, y pantalla en la medida en que la reescritura concreta intertextual en el centro de la producción textual se obscurece al reconocer el paralelo.

Sin embargo, el proceso se desarrolla a dos niveles, ya que el modelo es retomado al interior de la Recherche misma: los pasajes se conciben para significar a través de la activación de una especie de intertextua-

tar a Monsieur y a Madame como prototipos (véase más abajo) — aun cuando esconde los verdaderos procesos textuales implicados.

${ }^{12} \mathrm{Al}$ referirse al empleo de la palabra tante (término peyorativo para "homosexual" $\longrightarrow$ invertido, término preferido de Proust), este autor caracteriza su trabajo como su versión de la Comedia Humana. Después de constituirse como el arquetipo Guermantes, Saint-Loup eventualmente se convierte en el arquetipo tante $-\mathrm{y}$ el Narrador, describiendo a su amigo-vuelto-inverti puede demostrar sus finos poderes de observación. 
lidad intratextual en la que varios episodios basados en sutiles observaciones se conectan en una red de significados - con un doble movimiento hacia delante y hacia atrás, a la vez retrospección y anticipación. De esta manera, si en efecto la supresión de color tiene que ver con el secreto que Charlus tan obviamente carga, eventualmente el secreto se volverá, al menos para el Narrador, eminentemente descifrable. Y lo será a plena luz, tanto que le será difícil entender por qué los otros no ven la verdad acerca de Charlus:

Et pourtant, même sous les couches d'expressions différentes, de fards et d'hypocrisie qui le maquillaient si mal, le visage de M. de Charlus continuait à taire à presque tout le monde le secret qu'il me paraissait crier" (III, 731, énfasis agregado). ${ }^{13}$

De nueva cuenta, un elemento imperceptible es visible solo para el Narrador; se trata de una variante, que parte del conocimiento, del primer retrato de Charlus y de su "imperceptible" mancha roja. Al contrastar el claro significado que el rostro del Barón tiene para él y la ceguera de la gente a su alrededor, el Narrador pone de hecho de manifiesto su propia capacidad de ver e interpretar lo que percibe. Las Mémoires, como intertexto, quedan en un segundo plano: el recorrido puede llevar al retrato de Monsieur, pero lo hace a través de una conexión intratextual con la Recherche misma. Esta estrategia intratextual se torna sin cesar evidente en la Recherche con el Narrador (y por su intermediación con Proust), delegando ocasionalmente los poderes de observación del primero en algunos otros personajes relevantes de la obra.

\section{Una estrategia proliferante}

Entre los personajes de la Recherche, la duquesa de Guermantes (Oriane) y Swann juegan un papel mayor. Swann es especialmente central en

${ }^{13}$ Tales pasajes ayudan a aclarar la dialéctica del "clóset", en lo que Eve Sedgwick llamó "Epistemología del clóset" en su importante estudio que lleva ese título. Aun cuando los homosexuales "entran en el clóset" para esconderse, el último capitulo de Sedgwick se intitula "Proust y el espectáculo del clóset" (Sedgwick, 213-251). Y la reacción del Narrador evoca tal espectáculo. 
el proceso de construcción de la identidad, en particular en los paralelismos que se establecen a nivel textual entre él y Charlus.

Swann es algo así como un dandy, con gustos refinados y un toque de maneras pasadas de moda. El seguir posando su sombrero en el piso, que es presentado como un hábito tradicional, permite que el lector vislumbre un detalle colorido y que el Narrador construya, por lo menos para dos de sus principales personajes, un ethos de aguda perceptibilidad física y a la vez intelectual:

Serré dans une redingote gris perle, qui faisait valoir sa haute taille, svelte, ganté de gants blancs rayés de noir, il portait un tube gris d'une forme évasée que Delion ne faisait plus que pour lui, pour le prince de Sagan, pour M. de Charlus, pour le marquis de Modène, pour M. Charles Haas et pour le compte Louis de Turenne. [...] C'est ainsi encore que son chapeau, que, selon une habitude qui tendait à disparaître, il posa par terre à côté de lui, était doublé de cuir vert, ce qui ne se faisait pas d'habitude mais parce que c'était (à ce qu'il disait) beaucoup moins salissant, en réalité ${ }^{14}$ parce que c'était fort seyant (II, 866-867).

Algo que estaba oculto es aquí revelado y deviene altamente significativo a través del juego entre colores, tan vinculado con la identidad y tan persistente en la Recherche. ${ }^{15}$ Un contexto específico es creado por

${ }^{14}$ La edición de 1954 tiene un comentario de interés entre paréntesis: "(mais il ne le disait pas)" después de "en réalité" (II, 579).

${ }^{15}$ Los colores y las diversas oportunidades que ofrecen para el juego de palabras, están implicados en la actividad de identidad-producción en la que la Recherche constantemente se compromete. En sus inicios, los Guermantes están emparentados con Geneviève de Brabant, no solo genealógicamente, sino en el origen mismo de sus nombres: "[C]haque fois que je pensais à eux [el duque y la duquesa], je me les représentais tantôt en tapisserie, comme était la comtesse de Guermantes dans le 'Couronnement d'Esther' de notre église, tantôt de nuances changeantes, comme était Gilbert le Mauvais dans le vitrail où il passait du vert chou au bleu prune, selon que j'étais encore à prendre de l'eau bénite ou que j'arrivais à nos chaises, tantôt tout à fait impalpables comme l'image de Geneviève de Brabant, ancêtre de la famille de Guermantes, que la lanterne magique promenait sur les rideaux de ma chambre ou faisait monter au plafond - enfin toujours enveloppés du mystère des temps mérovingiens et baigant, comme dans un coucher de soleil, dans la lumière orangée qui émane de cette syllabe: 'antes"” (I, 169). "Gilbert le Mauvais" está relacionado, por la forma de su apodo, con la Duquesa, que el Narrador vio por primera vez en la capilla de los Guermantes, en la iglesia en la que ella lleva una corbata mauve (la corbata relaciona a ella con su cuñado, Charlus, y el color mauve de 
los nombres, con el efecto metaléptico ${ }^{16}$ de Charles Haas, el modelo para Swann, que está presente en la ficción y el nombre Turenne que remite al siglo XVII. ${ }^{17}$ No solamente el lector está dotado de información, sino que el detalle constituye otra oportunidad para confirmar la escrutadora mirada de la duquesa de Guermantes:

Comme c'est bien de faire doubler son chapeau de vert, dit la duchesse $\grave{a}$ qui rien n'échappait. D'ailleurs, en vous, Charles, tout est joli, aussi bien ce que vous portez que ce que vous dites, ce que vous lisez et ce que vous faites (II, 871, énfasis agregado).

Nada escapa a su mirada — un eco de la perceptibilidad del Narrador. Pero digamos que el elogio es mutuo. Charles Swann responderá de la misma manera al observar las joyas de la duquesa de Guermantes: "Quels magnifiques rubis!", con lo que esta advertirá que Swann reconoce lo auténtico cuando lo ve: “Ah! Mon petit Charles, au moins on voit que vous vous y connaissez, vous n'êtes pas comme cette brute de Monserfeuil qui me demandait s'ils étaient vrais" (871).

Estos intercambios son altamente significativos para la presente discusión, en la medida en que redoblan de dos formas distintas el ethos del Narrador. Por un lado, ambos personajes se presentan como agudos observadores. Nada escapa a la duquesa; la autenticidad de sus joyas no es ignorada por Swann - con el giro añadido de que ambos se reconocen mutuamente por lo que son, acuciosos observadores y testigos confiables. En esta medida se convierten, en un primer sentido, en dobles del Narrador porque, como él, ven a través de las cosas. Pero considerados conjuntamente también repiten las coloridas conexiones que hizo el Narrador cuando Charlus apareció por primera vez. Charles es, sin duda, una especie de álter ego de Charlus, no solo por la similitud fónica entre Charles y Charlus, o porque, conforme progresa la novela,

la corbata, con la ascendencia de los Guermantes, a través de Gilbert le mauvais, sin embargo, paradójicamente, se nos dice que la corbata es demasiado nueva.

${ }^{16}$ Sobre la metalepsis en narratología y en teoría de la ficción, véase Gérard Genette, Métalepse, De la figure à la fiction.

${ }^{17}$ Una metonimia de sastrería sitúa a Swann a la par con la contraparte de su "vida real" y con un aristócrata del siglo XVII, volviéndolo aquí y en todas partes un doble funcional de Charlus. 
tanto lo judaico como lo homosexual emergen como dos identidades metonímicas explícitamente paralelas, que empiezan siendo secretas pero gradualmente son reveladas. Tampoco es solo porque entre el forro verde de su sombrero, descubierto cuando la enfermedad había alterado considerablemente sus rasgos faciales, y el enrojecimiento resultado de la misma enfermedad, que desfiguró su nariz haciéndolo lucir como un viejo hebreo, Swann adopta, en cierta forma, los dos colores que atestiguan la supresión de color en la indumentaria de Charlus (una clave de su identidad, de la misma manera como la nueva nariz de Swann lo es para la suya). ${ }^{18}$ Es más específicamente porque, tanto Oriane de Guermantes como Charles Swann, perciben ya sea el color invisible (el forro verde) o la verdad que entraña el color (las joyas) — repitiendo así la propia hazaña del Narrador de percibir lo imperceptible, a pesar de que la revelación se demore, la verdad que entraña la percepción, la verdad intertextual de la identidad. Y la perceptibilidad del Narrador específicamente se enfatizó a través de su interpretación de la ausencia de color en el atuendo del Barón, mediante la coincidencia del hilo verde y la imperceptible mancha roja.

\section{De vuelta con el Narrador}

Proust ancla los poderes de discernimiento de su Narrador (que serán reafirmados en las páginas finales de la novela, y se orientan hacia la capacidad del Narrador para realizar su ser y vocación como escritor y así devenir, en efecto, el Narrador) en la conexión externa con el retrato de Monsieur de Saint-Simon. Pero no se olvidó de añadir ciertas referencias intratextuales sobre la construcción del ethos. La perceptibilidad corre en la sangre. La sostenida relación metonímica entre judíos

\footnotetext{
${ }^{18}$ El último avatar de Swann, como un antiguo profeta hebreo, ha sido tan a menudo comentado que se vuelve innecesario hacerlo aquí, excepto por la conexión del ineludible enrojecimiento de su nariz, que afirma el contraste con la imperceptible mancha roja que el Narrador discierne en la de otra manera austera corbata de Charlus: "le nez de polichinelle de Swann, longtemps résorbé dans un visage agréable, semblait maintenant énorme, tuméfié, cramoisi, plutôt celui d'un vieil Hébreu que d'un curieux Valois" (III, 89). A propósito de todo este pasaje, véase Zoberman, "Et Proust créa le Juif", 100-101.
} 
y homosexuales, que se vuelve explícita después de que el Narrador descubre a Charlus e identifica al homosexual, ha sido comentada por varios analistas. ${ }^{19}$ Ello también lleva a una genealogía de la perceptibilidad. Es muy probable que el lector proustiano recuerde que en los pasajes tempranos de Du côté de chez Swann se muestra la capacidad del abuelo para identificar judíos sin importar que tan bien se las arreglen para pasar desapercibidos:

Avant de les avoir vus, rien qu'en entendant leur nom qui, bien souvent, n'avait rien de particulièrement israélite, il devinait non seulement l'origine juive de ceux de mes amis qui l'étaient en effet, mais même ce qu'il $\mathrm{y}$ avait quelquefois de fâcheux dans leur famille $(\mathrm{I}, 90){ }^{20}$

Con semejante mago como abuelo, tan dado a atribuir identidades, no debería sorprender la capacidad de observación del Narrador. Este último, a su vez, pone de manifiesto las capacidades de discernimiento de otros personajes por medio de un vínculo intratextual con al menos un episodio previo. Y el Narrador convierte tales secuencias en interpretantes $^{21}$ sucesivos y acumulativos. Por consiguiente, cuando explica cómo solo se requiere un elemento revelador para dotar de conciencia la interpretación - que aunque obvia podría hasta entonces carecer de coherencia-, el Narrador está de hecho reuniendo todos los episodios significativos, de la misma manera como Proust vincula la Recherche con trabajos literarios más tempranos, en especial de autores franceses del siglo XVII. La comparación con una mujer preñada, en cuyo embarazo, por más que sea visible, uno no repara sino hasta que alguien llama nuestra atención, permite que el Narrador justifique su recién funda-

${ }^{19}$ Véanse Bem, Kristeva, Zoberman ("Et Proust créa le Juif”, "Prototypes chez Proust"), entre otros.

${ }^{20} \mathrm{Y}$ lo que el abuelo tararea o canta son melodías y palabras de la ópera La Juive, de Scribe y Halévy, de la que también procede la línea "Rachel, quand du Seigneur", broma recurrente sobre el personaje de Rachel con quien Gilberte es tan paradójicamente comparada.

${ }^{21}$ En el empleo que hace del término Michael Riffaterre (que desarrolla, en particular, en "Sémiotique intertextuelle") más que en el de Pierce (sobre el que Riffaterre, en efecto, se basa). 
mentado conocimiento así como la hasta entonces inexplicable ceguera, y que concluya:

De plus, je comprenais maintenant pourquoi tout à l'heure, quand je l'avais vu sortir de chez Mme de Villeparisis [una referencia a un pasaje anterior p. 6], j'avais pu trouver que M. de Charlus avait l'air d'une femme: c'en était une! (III, 16).

El misterio que generó la primera aparición de M. de Charlus en la novela lo resuelve finalmente el Narrador, quien por fin es capaz de presenciar una escena reveladora que lo lleva a articular la verdad acerca de Charlus - una verdad que siente estar cada vez más expuesta a la vista de todos, lo que lo lleva a preguntarse cómo es posible que los otros no la vean tan claramente. Pero lo que lo vuelve el testigo confiable que él/ Proust sostiene ser, es en realidad una construcción textual, o más bien intertextual, que conduce de un hilo verde y una mancha roja a un forro verde, auténticos rubíes y una nariz enrojecida por la arterioesclerosis y, por último, a una epifanía: si la nariz de Pulcinella de Swann lo revela como un hebreo arquetípico, Charlus se convierte en el Charlus, la tante, el homosexual. También vale la pena señalar que el color verde había sido asociado a un antepasado masculino de los Germantes, Gilbert le Mauvais ("Gilbert le Mauvais, dans le vitrail où il passait du vert chou au bleu prune selon que j'étais encore à prendre de l'eau bénite ou que j'arrivais à nos chaises" [I; 69]), ${ }^{22}$ y que el rojo es el color del vestido que lleva la duquesa durante su conversación con Swann.

Dada la teoría desarrollada al principio de Sodome et Gomorrhe, resulta bastante apropiado que el rojo y el verde de Charlus sean compartidos entre un personaje masculino y otro femenino al final de Le Côté de Guermantes y que debamos tener, unas cuantas páginas más adelante, en Sodome et Gomorrhe, como título del capítulo I: "première apparition des hommes-femmes", primera aparición de los hombres-mujer, esto es, de hombres que son mujeres.

De esta manera, los colores, (des)ordenados de acuerdo con el género, contribuyen a reforzar el poder de observación del Narrador, en especial

\footnotetext{
${ }^{22}$ Véase el contexo de este pasaje supra, nota 15.
} 
conforme va aprendiendo a leer los signos de identidad. Del desconcertado Narrador que advierte detalles que literalmente no son visibles, a Charles Swann y Oriane de Guermantes que son capaces de penetrar secretos que están a plena vista y de regreso al Narrador que ahora puede equiparar su interpretación con su agudo sentido de observación, la Recherche construye un ethos de perspicacidad, ingenio y visión a través de la escritura de estrategias que tienen que ver tanto con la validación intertextual como con la credibilidad individual (o incluso hasta más).

\section{REFERENCIAS}

BEM, Jeanne, "Le Juif et l'homosexuel dans À la recherché du temps perdu. Fonctionnements textuels", Littérature, 37, 1980, 100-112.

Compagnon, Antoine, "La Dernière Victime du narrateur", Critique, 598, 1997, 131-146.

De Ley, Herbert, Marcel Proust et le duc de Saint-Simon, Urbana / London, University of Illinois Press, 1966.

De MAN, Paul, Blindness and Insight: Essays in the Rhetoric of Contemporary Criticism, New York, Oxford University Press, 1970.

Deleuze, Gilles, Proust et les signes [1964], Paris, Presses Universitaires de France, 1970.

Faret, Nicolas, L'Honnête Homme, ou l'art de plaire à la Court, Paris, Du Bray, 1630.

Genette, Gérard. Métalepse. De la figure à la fiction, Paris, Le Seuil, 2002 (Poétique).

Hepp, Noémi, "Le XvII siècle de Marcel Proust dans La Recherche du temps perdu", Travaux de Linguistique et de Littérature, 17/2, 1974, 121-144.

Jullien, Dominique, Proust et ses modèles, Paris, José Corti, 1989.

Kristeva, Julia, Proust. Questions d'identité, Oxford, Legenda, 1998 (European Humanities Research Centre, Special Lecture Series, 1).

La Bruyère, Jean de, Les Caractères, R. Garapon (ed.), Paris, Garnier, 1962.

Proust, Marcel, À la recherche du temps perdu, edición bajo la dirección de Jean Yves Tadié, t. I, Paris, Gallimard, 1987; t. II y III, 1988; t. IV, 1989 (Bibliothèque de la Pléiade).

Proust, Marcel, À la recherche du temps perdu, Pierre Clarac y Alain Ferré (eds.), t. II, Paris, Gallimard, 1954 (Bibliothèque de la Plèiade).

RifFATERre, Michael, "Sémiotique intertextuelle: l'interprétant", Revue d'esthétique, 1-2, 1979, 128-150. 
SAInt-Simon, Mémoires, Yves Coirault (ed.), t. II, Paris, Gallimard, 1983; t. VIII, 1983-1988 (Bibliothèque de la Pléiade).

Sedgwick, Eve Kosofsky, The Epistemology of the Closet, Los Angeles, University of California Press, 1990.

Zoberman, Pierre, "Et Proust créa le Juif", Le Discours et la Langue. Revue de Linguistique Française et d'Analyse du Discours, 2009-2010, 1-2, 93 104.

Zoberman, Pierre, "Prototypes chez Proust: une recherche à corps perdu", Itinéraires. Littérature, Textes, Cultures, 2009, 1, 23-41. 